\title{
Magnetic properties of Dy-diffused Nd-Fe-B powder prepared by crystallization from amorphous state
}

\author{
Hirotoshi Fukunaga, Ikuo Yamamoto, ${ }^{a)}$ Masaki Nakano, and Takeshi Yanai \\ Graduate School of Engineering, Nagasaki University, Nagasaki 852-8521, Japan
}

(Presented 1 November 2011; received 30 September 2011; accepted 8 December 2011; published online 12 March 2012)

\begin{abstract}
The method of enhancing coercivity of isotropic Nd-Fe-B flakes using simultaneous diffusion of Dy and crystallization from the amorphous state was also applied to $\mathrm{Nd}-\mathrm{Fe}-\mathrm{B}$ powders, in this study. Metal Dy coating was synthesized on amorphous $\mathrm{Nd}_{2.36} \mathrm{Fe}_{14} \mathrm{~B}_{1.05}$ powders by the vapor deposition method, and the powders were crystallized by a flash annealing. Simultaneous diffusion of Dy from their surfaces using the crystallization enhanced the coercivity of isotropic $\mathrm{Nd}-\mathrm{Fe}-\mathrm{B}$ powders. The coercivity $H_{\mathrm{c}}$ increased and the remanence $M_{\mathrm{r}}$ decreased nearly linearly with the amount of Dy, and the coating of $4.5 \mathrm{wt} . \%$ Dy resulted in $25.3 \%$ increase in $H_{\mathrm{c}}$ and $6.5 \%$ decrease in $M_{\mathrm{r}}$. The decrease in $M_{\mathrm{r}}$ of the Nd-Fe-B powders is nearly the same in magnitude to that previously reported for $\mathrm{Nd}_{2.36} \mathrm{Fe}_{14} \mathrm{~B}_{1.05}$ flakes, which suggests that the method of Dy diffusion using crystallization from amorphous state is applicable to $\mathrm{Nd}-\mathrm{Fe}-\mathrm{B}$ amorphous powders as well as flakes. The energy-dispersive X-ray spectroscopy revealed that Dy coating is inhomogeneous, and a further enhancement in $H_{\mathrm{c}}$ is expected by improving the coating method. (C) 2012 American Institute of Physics. [doi:10.1063/1.3679422]
\end{abstract}

\section{INTRODUCTION}

$\mathrm{Nd}-\mathrm{Fe}-\mathrm{B}$-based magnets are used widely because of their highest energy product at room temperature. Their coercivity $H_{\mathrm{c}}$ is, however, lost drastically at temperatures exceeding $100^{\circ} \mathrm{C}$ because of the low Curie temperature $\left(313{ }^{\circ} \mathrm{C}\right)$ of $\mathrm{Nd}_{2} \mathrm{Fe}_{14} \mathrm{~B}$. ${ }^{1}$ This disadvantage can be overcome by substituting a part of $\mathrm{Nd}$ in $\mathrm{Nd}_{2} \mathrm{Fe}_{14} \mathrm{~B}$ by a heavy rareearth (RE) element such as Dy or $\mathrm{Tb}$, because $\mathrm{Dy}_{2} \mathrm{Fe}_{14} \mathrm{~B}$ and $\mathrm{Tb}_{2} \mathrm{Fe}_{14} \mathrm{~B}$ have high anisotropy fields even at a high temperature. ${ }^{1}$ On the other hand, the limited supplies of heavy rareearth elements ${ }^{2}$ and the small magnetization values of $\mathrm{Dy}_{2} \mathrm{Fe}_{14} \mathrm{~B}$ and $\mathrm{Tb}_{2} \mathrm{Fe}_{14} \mathrm{~B}$ raise the strong requirement for suppression of consumption of heavy RE resources. From the above point of view, the enhancement of $H_{\mathrm{c}}$ by the diffusion of a heavy RE element from their surfaces have been reported for sintered Nd-Fe-B magnets ${ }^{3,4}$ and MQ3 powders. ${ }^{5}$ The enhancement of $H_{\mathrm{c}}$ due to the diffusion of $\mathrm{Nd}-\mathrm{Cu}$ have been also reported for HDDR Nd-Fe-B powders. ${ }^{6,7}$

The enhancement of $H_{\mathrm{c}}$ is effective in improving the thermal stability of $\mathrm{Nd}-\mathrm{Fe}-\mathrm{B}$ anisotropic bonded magnets. ${ }^{6}$ Therefore, it is expected that the above diffusion technique can be used for improving the thermal stability of isotropic bonded magnets used at a relatively high temperature. In the methods mentioned above, however, the diffusion was carried out at a high temperature and/or for a long time. When such an annealing is applied to isotropic Nd-Fe-B powders crystallized from the amorphous state, it would degrade their magnetic properties because of grain growth. Thus, for isotropic Nd-Fe-B magnets, we proposed a simultaneous diffusion of Dy with crystallization from the amorphous state and confirmed that Dy coated on a surface of amorphous

\footnotetext{
a) Author to whom correspondence should be addressed. Electronic mail: bb52111250@cc.nagasaki-u.ac.jp.
}

$\mathrm{Nd}-\mathrm{Fe}-\mathrm{B}$ flakes diffuses in the flakes in extremely short time due to crystallization, and that $H_{\mathrm{c}}$ is improved without remarkable reduction of remanence. ${ }^{8}$ For applying this method to isotropic resin-bonded magnets, we have to coat Dy on the surface of amorphous powders rather than flakes. Thus, we needed to change the coating method (PLD) adopted in the previous study, ${ }^{8}$ because coating of powders would be difficult compared with that for flakes.

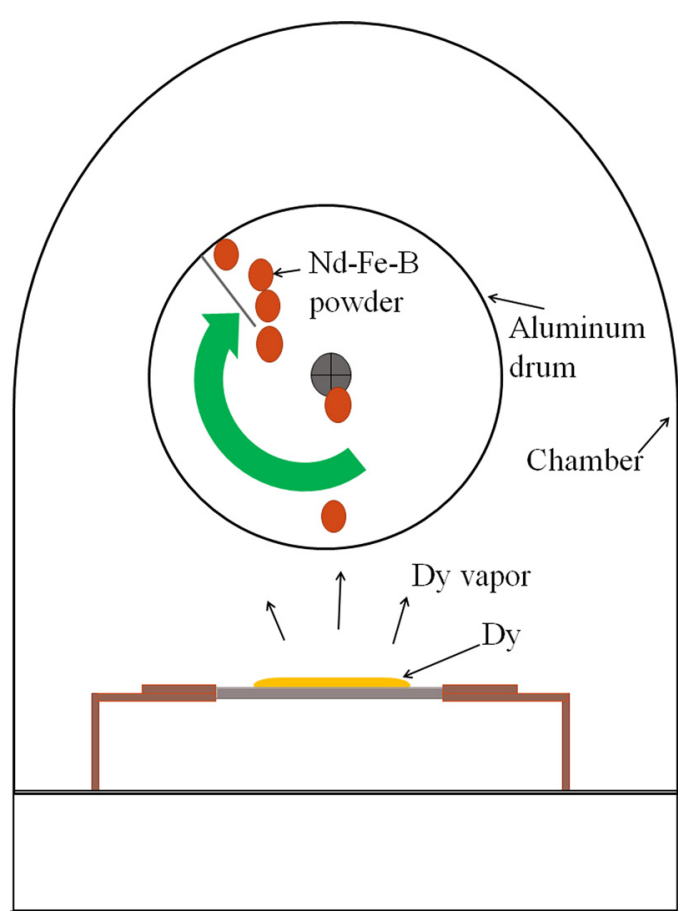

FIG. 1. (Color online) Schematic representation of vapor deposition apparatus used in this study. 


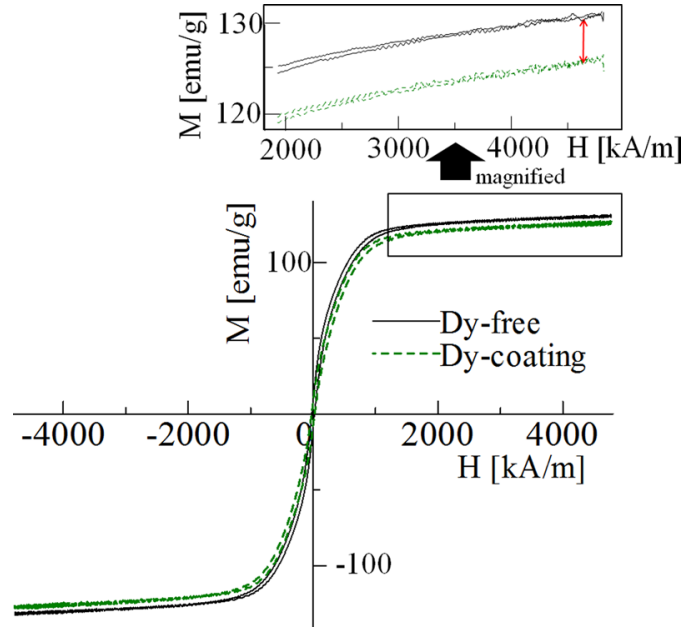

FIG. 2. (Color online) Magnetization curves of amorphous powders before and after Dy-coating. The inset indicates the magnetization curves under high field. The amount of coated Dy was determined from the difference in magnetization values between amorphous powders with and without Dy-coating.

In this contribution, we prepared an apparatus for powder coating, and applied the above-mentioned diffusionmethod to amorphous Nd-Fe-B powders prepared from amorphous flakes. The relationship between the amount of coated Dy and magnetic properties was studied. Consequently, the validity of the method principle proposed previously was confirmed for amorphous Nd-Fe-B powders.

\section{EXPERIMENTAL PROCEDURE}

Amorphous $\mathrm{Nd}_{2.36} \mathrm{Fe}_{14} \mathrm{~B}_{1.05}$ powders, with particle size in the range of $180-250 \mu \mathrm{m}$ were prepared by pulverizing the amorphous flakes followed by classification. Subsequently, metal Dy coating was synthesized on amorphous powders with a vapor deposition apparatus shown in Fig. 1. The amorphous $\mathrm{Nd}_{2.36} \mathrm{Fe}_{14} \mathrm{~B}_{1.05}$ powders were set in a drum, $6.7 \mathrm{~cm}$ in diameter, rotating at the speed of $42 \mathrm{rpm}$. The powders dropped from the top of the drum to its bottom during the rotation of the drum and were coated with metal Dy evaporated from a $\mathrm{W}$ boat. The deposition time was set at 2.5-3.0 min. The amount of coated Dy was varied by changing the amount of Dy mounted on the boat. Subsequently, the Dy-coated powders were crystallized, and Dy was diffused simultaneously inside the powders by heating them

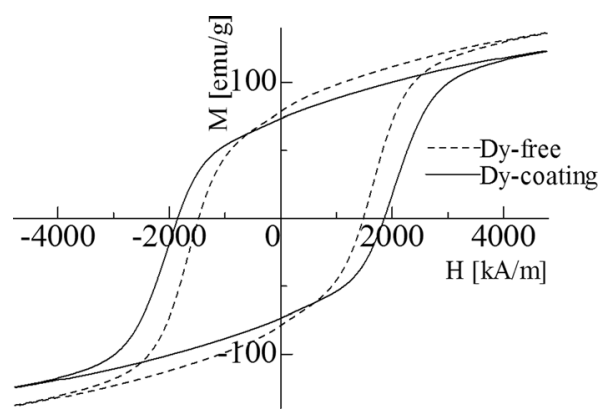

FIG. 3. Typical hysteresis loops of Nd-Fe-B powders crystallized with and without Dy-coating. The amount of coated Dy determined from the magnetization curve is $4.5 \%$.

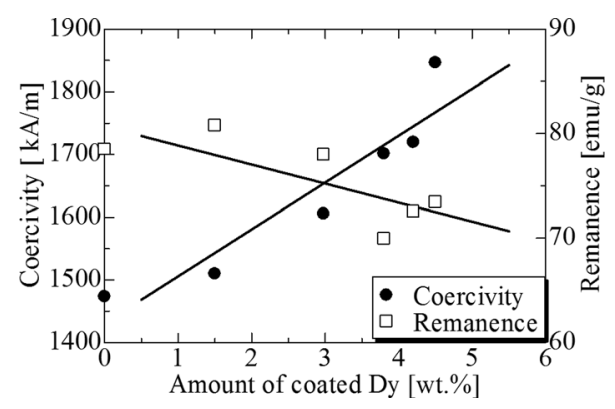

FIG. 4. Coercivity and remanence of Nd-Fe-B powder after crystallization as a function of the amount of coated Dy.

to $923 \mathrm{~K}$ followed by rapid cooling with an infrared furnace. The heating rate of the furnace was set at $462 \mathrm{~K} / \mathrm{min}$. Magnetic properties of the samples were measured with a vibrating sample magnetometer under the maximum applied field of $4.8 \mathrm{MA} / \mathrm{m}$. The amount of coated Dy was determined from the difference in magnetization values before and after coating as shown in Fig. 2. The distributions of $\mathrm{Nd}$, Dy, and $\mathrm{Fe}$ in Dy-coated powders were analyzed by energydispersive X-ray spectroscopy (EDS).

\section{RESULTS AND DISCUSSION}

Figure 3 shows typical hysteresis loops of Nd-Fe-B powders crystallized with and without Dy-coating. The amount of coated Dy determined from the magnetization curve is $4.5 \mathrm{wt} . \%$. The coercivity $H_{\mathrm{c}}$ increased by approximately $373 \mathrm{kA} / \mathrm{m}(25.3 \%)$ with a slight reduction in the remanence $M_{\mathrm{r}}$ of $5.1 \mathrm{emu} / \mathrm{g}(6.5 \%) . H_{\mathrm{c}}$ and $M_{\mathrm{r}}$ are plotted in Fig. 4 as a function of the amount of coated Dy. The increase in Dy increased $H_{\mathrm{c}}$ and decreased $M_{\mathrm{r}}$ nearly linearly to the amount of Dy. This result suggests that Dy-coating on amorphous NdFe-B powders followed by their crystallization enables us to improve $H_{\mathrm{c}}$ without remarkable reduction in $M_{\mathrm{r}}$.

The relationship between $H_{\mathrm{c}}$ and $M_{\mathrm{r}}$ is shown in Fig. 5 together with results obtained for $\mathrm{Nd}_{2.36} \mathrm{Fe}_{14} \mathrm{~B}_{1.05}$ flakes in the previous work. ${ }^{8}$ The decrease in $M_{\mathrm{r}}$ of the powders is nearly the same in magnitude to that reported previously for $\mathrm{Nd}_{2.36} \mathrm{Fe}_{14} \mathrm{~B}_{1.05}$ flakes, which suggests that the method of Dy diffusion using crystallization from amorphous state is applicable to $\mathrm{Nd}-\mathrm{Fe}-\mathrm{B}$ amorphous powders as well as flakes.

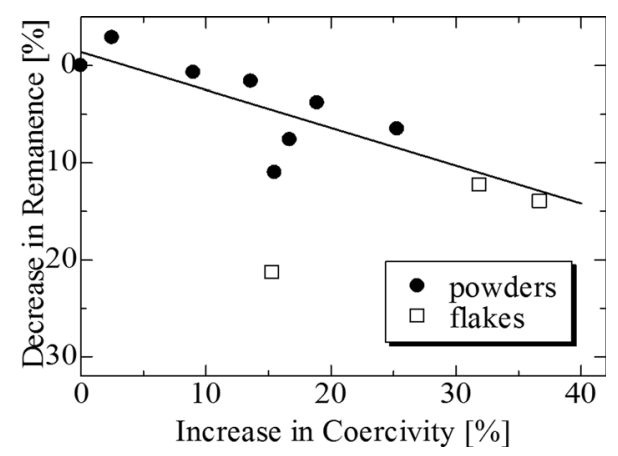

FIG. 5. Variation of coercivity and remanence of Nd-Fe-B powders crystallized after Dy-coating together with results for flakes reported previously (Ref. 8). 


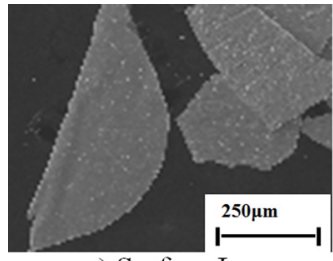

(a) Surface Image

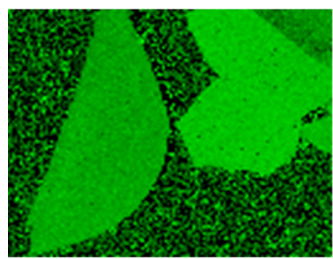

(c) $\mathrm{Fe}$

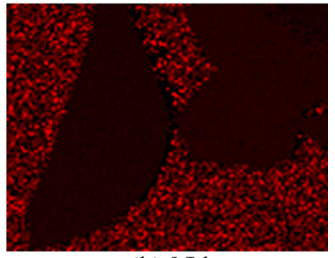

(b) $\mathrm{Nd}$

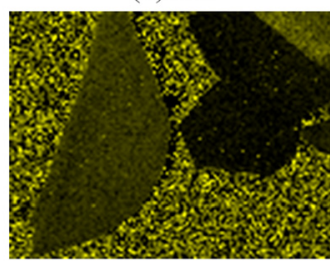

(d) Dy
FIG. 6. (Color online) SEM image and distributions of Nd, Fe, and Dy analyzed by EDS for Nd-Fe-B powders crystallized after Dy-coating. (a) Surface image, (b) Nd map, (c) Fe map, and (d) Dy map.

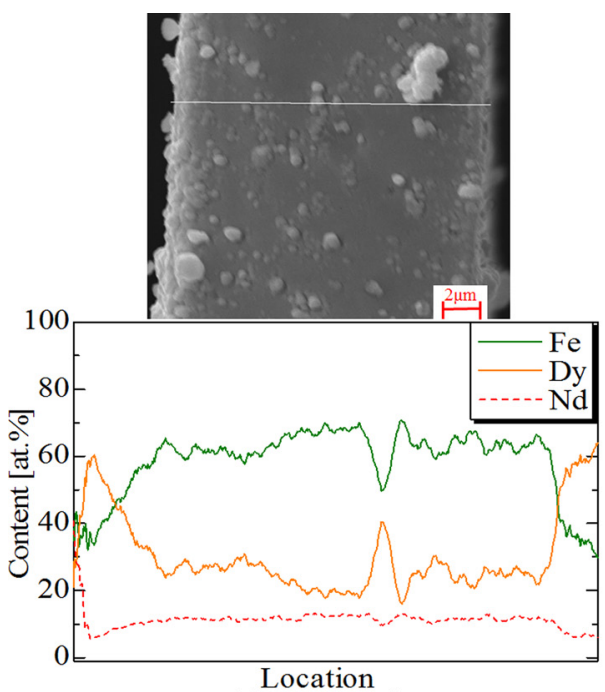

(a) cross section

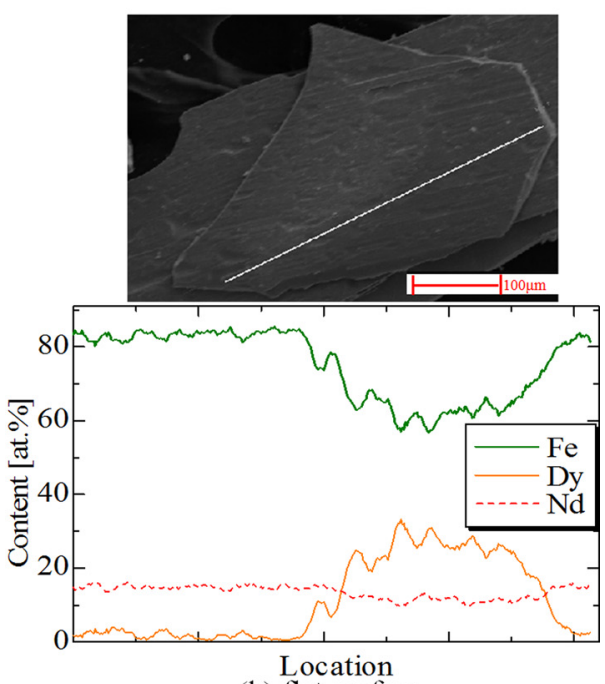

(b) flat surface

FIG. 7. (Color online) Results of EDS line analysis of Nd, Fe, and Dy contents for (a) cross section and (b) flat surface of crystallized Nd-Fe-B powders, together with the images of corresponding powders. The B content was neglected. The white lines in the images indicate the lines analyzed.
Figure 6 shows the distributions of Nd, Fe, and Dy analyzed by EDS for Dy coated powders after crystallization together with the surface image of the powders. The elemental maps (Figs. 6(b) and 6(d)) indicate that Dy coating was synthesized on the surfaces of powders.

The results of line analyses of $\mathrm{Nd}, \mathrm{Fe}$, and Dy contents are shown in Fig. 7 together with the surface images. As shown in Fig. 7(a), the cross section of the particles was also covered with Dy, which suggests that the coating method adopted in this investigation is useful to make Dy coating on powders obtained by pulverization of flakes.

As seen in Fig. 7(b), on the other hand, the observed particle is covered only partially with Dy. It was also observed that the ratio of Dy to $\mathrm{Nd}$ varies from particle to particle. These results suggest that the thickness of coated Dy is inhomogeneous. A further enhancement of $H_{\mathrm{c}}$ is expected by improving the coating method.

\section{CONCLUSIONS}

Isotropic amorphous $\mathrm{Nd}_{2.36} \mathrm{Fe}_{14} \mathrm{~B}_{1.05}$ powders prepared by pulverization of flakes were coated by Dy, and the coercivity was enhanced by utilizing simultaneous diffusion of Dy with the crystallization from the amorphous state. The coercivity $H_{\mathrm{c}}$ increased and the remanence $M_{\mathrm{r}}$ decreased nearly linearly with the amount of Dy. The coating of 4.5 wt. \% Dy resulted in 25.3\% increase of $H_{\mathrm{c}}$ and $6.5 \%$ decrease of $M_{\mathrm{r}}$. The decrease in $M_{\mathrm{r}}$ of the Nd-Fe-B powders in this investigation is nearly the same in magnitude to that reported previously for $\mathrm{Nd}_{2.36} \mathrm{Fe}_{14} \mathrm{~B}_{1.05}$ flakes, which suggests that the method of Dy diffusion using crystallization from amorphous state is applicable to Nd-Fe-B amorphous powders as well as flakes. The energy-dispersive X-ray spectroscopy revealed that Dy coating is inhomogeneous, and a further enhancement of $H_{\mathrm{c}}$ is expected by improving the coting method.

\section{ACKNOWLEDGMENTS}

We thank Dr. T. Iriyama of Daido Steel Co., Ltd. for kindly supplying the Nd-Fe-B flakes. This work was supported in part by the Ministry of Education Science Sports and Culture of Japan MESSC (JP) under a Grant-in-Aid (22360128).

${ }^{1}$ M. Sagawa, S. Hirosawa, H. Yamamoto, S. Fujimura, and Y. Matsuura, Jpn. J. Appl. Phys. 26, 785 (1987).

${ }^{2}$ J. Lifton, Proc. 21 st Workshop on Rare-Earth Permanent Magnets and their Applications, Bled, Slovenia, 2010, pp. 27-30.

${ }^{3}$ H. Nakamura, K. Hirota, T. Minowa, and M. Honshima, IEEE Trans. Magn. 42, 2909 (2006).

${ }^{4}$ D. S. Li, S. Suzuki, T. Kawasaki, and K. Machida, Jpn. J. Appl. Phys. 47, 7876 (2008).

${ }^{5}$ M. Komuro, Y. Satsu, H. Suzuki, Proc. 21st Workshop on Rare-Earth Permanent Magnets and their Applications, Bled, Slovenia, 2010, pp. 243-245.

${ }^{6}$ C. Mishima, K. Noguchi, M. Yamazaki, M. Mitarai, and Y. Honkura, Proc. 21 st Workshop on Rare-Earth Permanent magnets and their Applications, Bled, Slovenia, 2010, pp. 253-256.

${ }^{7}$ H. Sepehri-Amin, T. Ohkubo, T. Nishiuchi, S. Hirokawa, and K. Hono, Scripta Mater. 63, 1124 (2010).

${ }^{8}$ H. Fukunaga, Y. Sugimoto, M. Nakano, and T. Yanai, J. Appl. Phys. 109, 07A701 (2011) 